\title{
Reactivation of Hemophagocytic Lymphohistiocytosis Triggered by Antithymocyte Globulin
}

\author{
Kensuke Matsuda ${ }^{1}$, Kazuhiro Toyama ${ }^{2}$, Takashi Toya ${ }^{1}$, Masako Ikemura ${ }^{3}$, \\ Fumihiko Nakamura ${ }^{1}$ and Mineo Kurokawa ${ }^{1,2}$
}

\begin{abstract}
:
A 16-year-old boy with Epstein-Barr virus-associated hemophagocytic lymphohistiocytosis (HLH) underwent allogeneic hematopoietic stem cell transplantation after conditioning with fludarabine, melphalan, total body irradiation, and rabbit antithymocyte globulin (ATG). A severe, persistent infusion reaction occurred after the initial administration of ATG. Investigations showed a rapid increase in the levels of liver enzymes and ferritin, and the reactivation of HLH was confirmed by marked hemophagocytosis in the bone marrow. Treatment with pulse glucocorticoid therapy resulted in the improvement of HLH. This is the first case of HLH reactivation triggered by ATG. Physicians should therefore be cautious of HLH reactivation, especially when a severe and prolonged infusion reaction occurs.
\end{abstract}

Key words: antithymocyte globulin, Epstein-Barr virus, hemophagocytic lymphohistiocytosis, infusion reaction

(Intern Med 57: 583-586, 2018)

(DOI: 10.2169/internalmedicine.9226-17)

\section{Introduction}

Secondary hemophagocytic lymphohistiocytosis (HLH) is a hyper-inflammatory disorder that develops as a result of various infections and malignancies. The massive activation of macrophages and cytokine release are induced by uncontrolled infection and malignancies. The resultant immune dysregulation is believed to play a pivotal role in the pathogenesis of HLH (1). The most common cause of secondary HLH is Epstein-Barr virus (EBV) infection. The incidence of EBV-associated HLH (EBV-HLH) is especially high in Asia (2), which is explained by the high prevalence of EBV infection in Asian people and host immunogenetic factors. The clinical features of HLH include fever, hepatosplenomegaly, cytopenias, hyperferritinemia, elevated transaminases, high serum soluble interleukin-2 receptor (sIL-2R), and hemophagocytosis. The initial treatment for HLH is usually immunosuppressive therapy composed of corticosteroids with or without cyclosporine A (CsA) and etoposide (ETP) (3). Allogeneic hematopoietic stem cell transplantation (HSCT) is the only curative treatment.

Antithymocyte globulin (ATG) is a polyclonal antibody drug that is used in immunosuppressive therapy of HLH (4). ATG induces the lysis of T-cells and other target cells in a complement-dependent fashion and restores immune dysregulation, mainly through the depletion of T-cells $(5,6)$. Similarly to other antibody drugs, infusion reactions are a frequent adverse effect of ATG. An infusion reaction is an infusion-related side effect that manifests as transient fever, tachycardia, and rash (7). Although the pathogenesis of infusion reaction has not yet been well elucidated, previous reports have revealed that complement activation play a key role in infusion reactions (7). We herein describe a case of EBV-HLH reactivation which was triggered by an infusion reaction in a patient who was treated with ATG.

\section{Case Report}

A 16-year-old boy presented to our institute with persis-

\footnotetext{
${ }^{1}$ Department of Hematology and Oncology, Graduate School of Medicine, The University of Tokyo, Japan, ${ }^{2}$ Department of Cell Therapy and Transplantation Medicine, The University of Tokyo Hospital, Japan and ${ }^{3}$ Department of Pathology, Graduate School of Medicine, The University of Tokyo, Japan

Received: March 22, 2017; Accepted: July 25, 2017; Advance Publication by J-STAGE: December 8, 2017

Correspondence to Dr. Mineo Kurokawa, kurokawa-tky@umin.ac.jp
} 


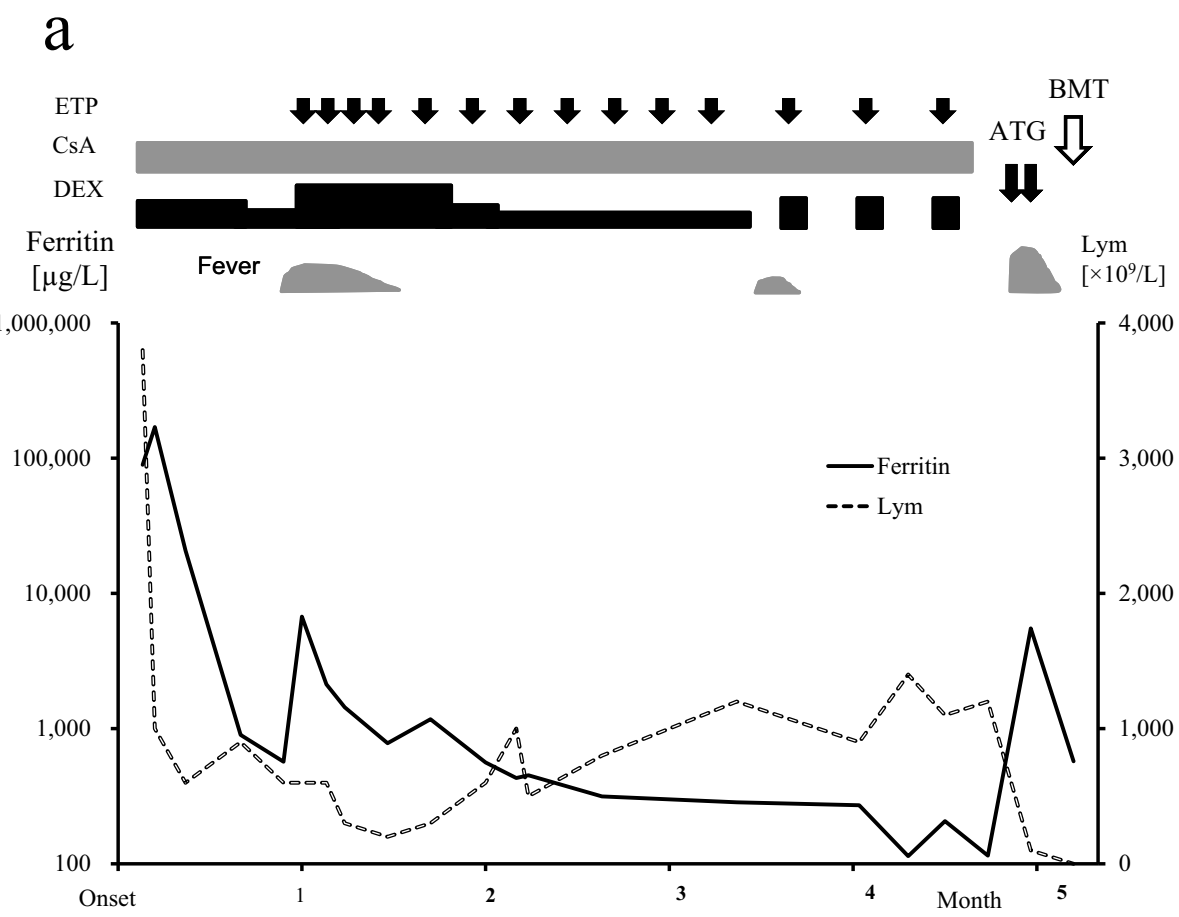

b

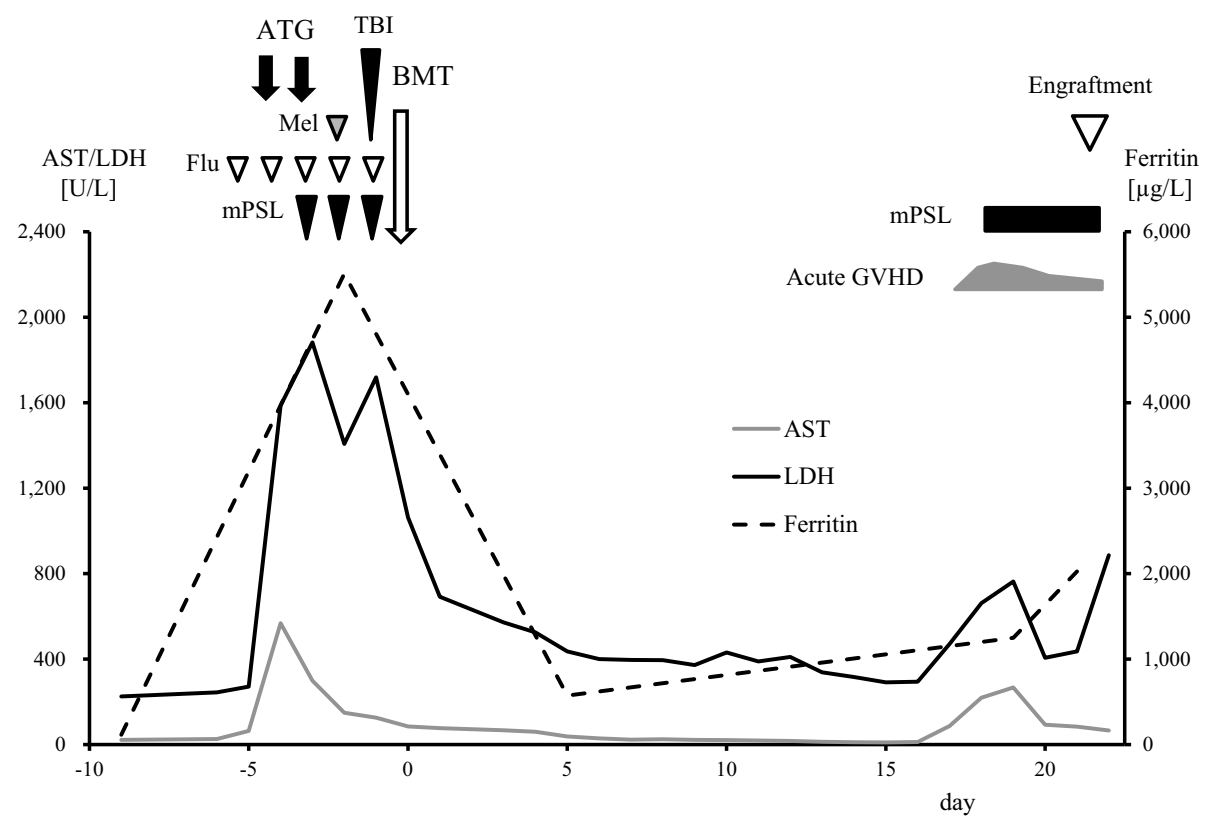

Figure. The clinical course. a: The overall clinical course from the onset of symptoms until bone marrow transplantation. b: The detailed clinical course from transplantation conditioning until neutrophil engraftment. Acute graft versus host disease (GVHD; grade II) was successfully treated with mPSL ( 2 mg/kg, daily). DEX: dexamethasone, CsA: cyclosporine A, ETP: etoposide, ATG: antithymocyte globulin, BMT: bone marrow transplantation, Lym: lymphocyte, Flu: fludarabine $\left(30 \mathrm{mg} / \mathrm{m}^{2} /\right.$ day, day -6 to -2), Mel: melphalan (140 mg/m², day -2), TBI: total body irradiation (4 Gy, day -1), mPSL: methyl-prednisolone, AST: aspartate aminotransferase, LDH: lactate dehydrogenase

tent fever following an episode of initial exposure to EBV. A physical examination revealed hepatosplenomegaly. Laboratory analyses revealed the following findings: hemoglobin, $132 \mathrm{~g} / \mathrm{L}$; platelet count, $41 \times 10^{9} / \mathrm{L}$; aspartate aminotransferase, 2,893 U/L; serum triglycerides, $3.0 \mathrm{mmol} / \mathrm{L}$; serum ferritin, 89,400 $\mu \mathrm{g} / \mathrm{L}$; and serum sIL-2R, 18,917 U/mL (Figure a). The patient's anti-viral capsid antigen (VCA)immunoglobulin $\mathrm{G}$ ( $\mathrm{IgG}$ ) titer was 1:40; there was no increase in the levels of VCA-IgM and EBV nucleoside antigen antibodies. The whole blood EBV-DNA load was 1,310 
copies per $\mu \mathrm{g}$ DNA. Bone marrow aspiration revealed hemophagocytosis by activated macrophages. Based on these findings, he was diagnosed with EBV-HLH. A pathological examination of the bone marrow aspirate revealed the accumulation of CD3-positive atypical lymphocytes, which were found to be positive for EBV-RNA by in situ hybridization (EBER-ISH). The treatment of HLH was initiated with dexamethasone and CsA, which resulted in defervescence and the normalization of the platelet count and liver enzyme levels. The addition of weekly intravenous ETP was required due to the recurrence of HLH during the attenuation of dexamethasone and CsA. The patient finally underwent bone marrow transplantation from a matched unrelated donor following reduced-intensity conditioning (RIC) with fludarabine $\left(30 \mathrm{mg} / \mathrm{m}^{2} /\right.$ day, day -6 to -2$)$, melphalan $\left(140 \mathrm{mg} / \mathrm{m}^{2}\right.$, day -2), total body irradiation (4 Gy, day -1), and rabbit ATG (Thymoglobulin ${ }^{\circledR}, 1.25 \mathrm{mg} / \mathrm{kg} /$ day, day $-5,-4$ ), as shown in Figure b. ATG was added to the RIC regimen with the aim of achieving T-cell depletion. Dexamethasone, CsA, and ETP were discontinued on days $-11,-7$, and -20 , respectively. On day -5 , premedication with methylprednisolone $(1 \mathrm{mg} / \mathrm{kg})$ was delivered prior to the initial ATG infusion; nevertheless, the patient developed high fever and tachycardia, which was consistent with a severe infusion reaction (grade 3 according to CTCAE version 4.0). The fever persisted through the following day, and laboratory analyses revealed an increase in the patient's aspartate aminotransferase $(568 \mathrm{U} / \mathrm{L})$ and ferritin $(5,510 \mu \mathrm{g} / \mathrm{L})$ levels. Bone marrow aspiration revealed marked hemophagocytosis, leading to the diagnosis of recurrent HLH. Pulse glucocorticoid therapy with methylprednisolone was initiated, which resulted in the clinical improvement of HLH. Bone marrow transplantation was performed as scheduled. Tacrolimus and short-term methotrexate were administered as prophylaxis against graft versus host disease (GVHD). On day 18, the patient developed grade 2 acute GVHD (skin, stage 0; liver, stage 1; gut, stage 1), which was successfully treated with methylprednisolone (2 mg/kg, daily). Simultaneous cytomegalovirus antigenemia was ameliorated with foscarnet (intravenous) from day 15 to 23. No other infections were recorded. Neutrophil engraftment with complete donor chimerism was achieved on day 22. On day 222, the patient's whole blood was negative for EBV-DNA. He has been relapse-free for 24 months since transplantation.

\section{Discussion}

This is the reported case of EBV-HLH reactivation immediately after the administration of ATG. Infusion reactionrelated symptoms are usually transient and disappear after the end of administration. In this case, however, the inflammatory symptoms persisted after the administration of ATG, despite the administration of an appropriate dosage of antiinflammatory drugs. The increase in the transaminase and ferritin levels on the following day suggested the recurrence of HLH. Bone marrow aspiration showed marked hemo- phagocytosis.

The mechanism of action of ATG is mainly dependent on T-cell depletion through complement-dependent cell lysis $(5,6)$. Complement activation induces the release of various chemical mediators, and results in an infusion reaction (7). Macrophage activation and the release of cytokines, which are strongly associated with the pathogenesis of HLH, are also mediated by complement activation (8). Thus, the relationship between the infusion reaction and the recurrence of HLH may be partly explained by the induction of complement activation following the administration of ATG. In this case, it is possible that ATG exerts its effect through complement activation, and that complement activation not only yielded an infusion reaction but also resulted in the recurrence of HLH. Previous reports on the induction of HLH by rituximab and infliximab may support this notion because these drugs have the potential to cause infusion reactions $(9,10)$.

It is also possible that the recurrence of HLH was induced by other causes. First, the recurrence of HLH might occur due to the discontinuation of combination therapy with DEX, CsA and ETP. However, there have been no reports on HLH reactivation shortly before transplantation under these circumstances. Furthermore, the patient had no symptoms and none of the patient's laboratory results suggested of the recurrence of HLH before the administration of ATG. Thus, a more reasonable explanation is that ATG triggered the recurrence of HLH. Second, the recurrence of HLH might be related to EBV reactivation because ATG is a well-known risk factor for viral reactivation (11). The latency period in our case was too short to support this notion. ATG-related EBV reactivation has thus far only been reported after transplantation in patients with lymphoproliferative disorder, with a median latency period of 71 days. Third, other preconditioning drugs, such as fludarabine could have influenced the recurrence of HLH.

In this case, although HLH was controlled after pulse glucocorticoid therapy, it is possible that the conditioning regimen, which included fludarabine, melphalan, and total body irradiation, also suppressed the progression of HLH because these treatments may have immunosuppressive effects.

In conclusion, physicians should be aware of the possibility of the immediate reactivation of HLH in patients who are treated with ATG, especially when a severe infusion reaction is seen.

The authors state that they have no Conflict of Interest (COI).

\section{References}

1. Chandrakasan S, Filipovich AH. Hemophagocytic lymphohistiocytosis advances in pathophysiology, diagnosis, and treatment. J Pediatr 163: 1253-1259, 2013.

2. Ishii E, Ohga S, Imashuku S, et al. Nationwide survey of hemophagocytic lymphohistiocytosis in Japan. Int J Hematol 86: 58-65, 2007. 
3. Imashuku S. Treatment of Epstein-Barr virus-related hemophagocytic lymphohistiocytosis (EBV-HLH); update 2010. J Pediatr Hematol Oncol 33: 35-39, 2011.

4. Jordan MB, Filipovich AH. Hematopoietic cell transplantation for hemophagocytic lymphohistiocytosis: a journey of a thousand miles begins with a single (big) step. Bone Marrow Transplant 42: 433-437, 2008.

5. Mohty M. Mechanisms of action of antithymocyte globulin: T-cell depletion and beyond. Leukemia 21: 1387-1394, 2007.

6. Ayuk FA, Fang L, Fehse B, et al. Antithymocyte globulin induces complement-dependent cell lysis and caspase-dependent apoptosis in myeloma cells. Exp Hematol 33: 1531-1536, 2005.

7. van der Kolk LE, Grillo-López AJ, Baars JW, et al. Complement activation plays a key role in the side-effects of rituximab treatment. Br J Haematol 115: 807-811, 2001.

8. Khan MA, Assiri AM, Broering DC. Complement and macro- phage crosstalk during process of angiogenesis in tumor progression. J Biomed Sci 22: 58, 2015.

9. Ramos-Casals M, Brito-Zerón P, López-Guillermo A, et al. Adult haemophagocytic syndrome. Lancet 383: 1503-1516, 2014.

10. Brito-Zerón $P$, Bosch $X$, Pérez-de-Lis $M$, et al. Infection is the major trigger of hemophagocytic syndrome in adult patients treated with biological therapies. Semin Arthritis Rheum 45: 391399, 2016.

11. Uhlin M, Wikell H, Sundin M, et al. Risk factors for Epstein-Barr virus-related post-transplant lymphoproliferative disease after allogeneic hematopoietic stem cell transplantation. Haematologica 99: 346-352, 2014.

The Internal Medicine is an Open Access article distributed under the Creative Commons Attribution-NonCommercial-NoDerivatives 4.0 International License. To view the details of this license, please visit (https://creativecommons.org/licenses/ by-nc-nd/4.0/).

(C) 2018 The Japanese Society of Internal Medicine Intern Med 57: 583-586, 2018 